OPEN ACCESS

Edited by:

Cristina Moglia,

University of Turin, Italy

Reviewed by:

Xuemin Xu,

University of Texas of the Permian

Basin, United States

Roland Brandt,

University of Osnabrück, Germany

*Correspondence:

Jochen H. Weishaupt

jochen.weishaupt@medma. uni-heidelberg.de

Specialty section

This article was submitted to

Neurodegeneration

a section of the journal

Frontiers in Neuroscience

Received: 11 April 2020

Accepted: 12 August 2020

Published: 11 September 2020

Citation:

Freischmidt A, Brenner $D$ Ludolph AC, Andersen PM and Weishaupt JH (2020) Commentary:

Effects of ALS-associated TANK

binding kinase 1 mutations on protein-protein interactions and kinase activity. Front. Neurosci. 14:551006 doi: 10.3389/fnins.2020.551006

\section{Commentary: Effects of ALS-associated TANK binding kinase 1 mutations on protein-protein interactions and kinase activity}

\author{
Axel Freischmidt ${ }^{1,2}$, David Brenner ${ }^{1,3}$, Albert C. Ludolph ${ }^{1,2}$, Peter M. Andersen ${ }^{4}$ and \\ Jochen H. Weishaupt ${ }^{1,3 *}$ \\ ${ }^{1}$ Department of Neurology, Ulm University, Ulm, Germany, ${ }^{2}$ German Center for Neurodegenerative Diseases (DZNE) Ulm, \\ UIm, Germany, ${ }^{3}$ Division for Neurodegenerative Diseases, Neurology Department, University Medicine Mannheim, Heidelberg \\ University, Mannheim, Germany, ${ }^{4}$ Department of Clinical Science, Neurosciences, Umeå University, Umeå, Sweden
}

Keywords: amyotrophic lateral sclerosis, ALS, frontotemporal dementia, FTD, TBK1, neuroinflammation, genetic counseling, genetic variants

\section{A Commentary on}

Effects of ALS-associated TANK binding kinase 1 mutations on protein-protein interactions and kinase activity

by Ye, J., Cheung, J., Gerbino, V., Ahlsén, G., Zimanyi, C., Hirsh, D., et al. (2019). Proc. Natl. Acad. Sci. U.S.A. 116, 24517-24526. doi: 10.1073/pnas.1915732116

\section{THE VAST MAJORITY OF TBK1 MISSENSE VARIANTS ARE OF UNKNOWN PATHOGENICITY}

TANK-binding kinase 1 (TBK1) is a serine/threonine kinase involved in the regulation of essential cellular functions, including innate immunity and selective autophagy (Helgason et al., 2013). Loss-of-function mutations have been demonstrated to cause amyotrophic lateral sclerosis (ALS) and frontotemporal dementia (FTD) (Cirulli et al., 2015; Freischmidt et al., 2015). Recently, Ye et al. (2019) reported functional effects of 25 TBK1 missense variants found in patients with ALS or FTD. They focus on impaired protein-protein interactions and substrate-specific defects in pathways such as innate immunity and autophagy. The authors describe that "TBK1 ALS mutations display a variety of defects" in different cellular pathways and conclude that "multiple defects can be caused by mutations from a single disease-associated gene but can lead to the common pathogenic outcome."

We would like to point out that the genetic link between TBK1 and neurodegeneration is largely based on Mendelian dominantly inherited deleterious loss-of-function mutations (such as splice site or frameshift mutations), which are enriched in ALS/FTD patients and result in a loss of expression of one TBK1 allele (Freischmidt et al., 2015). Importantly, several splice-site variants of TBK1 (Freischmidt et al., 2015) as well as an in-frame deletion of a single amino acid [TBK1 $\triangle$ E643; (Gijselinck et al., 2015)] have been shown to co-segregate with the disease over multiple generations. 
In stark contrast, rare or unique $T B K 1$ missense variants are also frequently detected in healthy controls (Cirulli et al., 2015; Freischmidt et al., 2015), although, overall, being significantly enriched in ALS/FTD patients compared to controls (Cirulli et al., 2015). However, the study by Cirulli et al. (2015) does not allow drawing conclusions about the pathogenicity of any specific TBK1 missense variant. Moreover, little or no data about co-segregation with disease are available with regard to the vast majority of these missense variants. The fact that an extremely rare or novel variant was detected in single or very few unrelated individuals [see e.g., (Freischmidt et al., 2017)] with a certain disease is by far not a proof of causality. Significant co-segregation with the disease (preferably in more than one family) or enrichment of the single, specific variant in patients compared to controls would provide a more solid body of evidence for causality of a specific variant. Hence, in our view (Freischmidt et al., 2017) and in accordance with the ACMG Standards and guidelines for classifying pathogenic variants (Richards et al., 2015) almost all missense variants in TBK1 must be considered "variants of unknown significance" (VUS). While a certain percentage of these TBK1 missense variants may of course be pathogenic, it is highly likely that some or even

\section{REFERENCES}

Cirulli, E.T., Lasseigne, B.N., Petrovski, S., Sapp, P.C., Dion, P.A., Leblond, C.S., et al. (2015). Exome sequencing in amyotrophic lateral sclerosis identifies risk genes and pathways. Science 347, 1436-1441. doi: 10.1126/science.aaa3650

Freischmidt, A., Müller, K., Ludolph, A.C., Weishaupt, J.H., and Andersen, P.M. (2017). Association of mutations in TBK1 with sporadic and familial amyotrophic lateral sclerosis and frontotemporal dementia. JAMA Neurol. 74, 110-113. doi: 10.1001/jamaneurol.2016.3712

Freischmidt, A., Wieland, T., Richter, B., Ruf, W., Schaeffer, V., Müller, K., et al. (2015). Haploinsufficiency of TBK1 causes familial ALS and fronto-temporal dementia. Nat. Neurosci. 18, 631-636. doi: 10.1038/nn.4000

Gijselinck, I., Van Mossevelde, S., van der Zee, J., Sieben, A., Philtjens, S., Heeman, B., et al. (2015). Loss of TBK1 is a frequent cause of frontotemporal dementia in a Belgian cohort. Neurology 85, 2116-2125. doi: 10.1212/WNL.0000000000002220

Helgason, E., Phung, Q.T., and Dueber, E.C. (2013). Recent insights into the complexity of Tank-binding kinase 1 signaling networks: the emerging role of cellular localization in the activation and substrate specificity of TBK1. FEBS Lett. 587, 1230-1237. doi: 10.1016/j.febslet.2013.01.059

Richards, S., Aziz, N., Bale, S., Bick, D., Das, S., Gastier-Foster, J., et al. (2015). Standards and guidelines for the interpretation of sequence variants: a joint most of them represent weak risk factors, or are unrelated to ALS/FTD causation.

Therefore, referring to TBK1 missense variants detected in ALS patients as (pathogenic) "ALS mutations" (Ye et al., 2019) is misleading. For the unclear causality, a correlation between different distinct downstream effects of TBK1 variants and ALS/FTD is currently impossible.

Consequently, while the value of the study by Ye et al. (2019) for the structural biological understanding of TBK1 function is undisputable, their conclusion that impairment of various different cell biological TBK1 functions may result in ALS/FTD is based on analyses of "variants of unknown significance" and is currently not supported by genetic evidence. We think that this should be taken into careful consideration when interpreting experimental results based on TBK1 missense variants, but also with regard to the genetic counseling of affected individuals and their families.

\section{AUTHOR CONTRIBUTIONS}

All authors listed have made a substantial, direct and intellectual contribution to the work, and approved it for publication.

consensus recommendation of the American College of Medical Genetics and Genomics and the Association for Molecular Pathology. Genet. Med. 17, 405-424. doi: 10.1038/gim.2015.30

Ye, J., Cheung, J., Gerbino, V., Ahlsén, G., Zimanyi, C., Hirsh, D., et al. (2019). Effects of ALS-associated TANK binding kinase 1 mutations on protein-protein interactions and kinase activity. Proc. Natl. Acad. Sci. U.S.A. 116, 24517-24526. doi: 10.1073/pnas.19157 32116

Conflict of Interest: The authors declare that the research was conducted in the absence of any commercial or financial relationships that could be construed as a potential conflict of interest.

The handling editor declared a past co-authorship with one of the authors PA.

Copyright (C) 2020 Freischmidt, Brenner, Ludolph, Andersen and Weishaupt. This is an open-access article distributed under the terms of the Creative Commons Attribution License (CC BY). The use, distribution or reproduction in other forums is permitted, provided the original author(s) and the copyright owner(s) are credited and that the original publication in this journal is cited, in accordance with accepted academic practice. No use, distribution or reproduction is permitted which does not comply with these terms. 